\title{
Study towards diversity oriented synthesis of optically active substituted cyclopentane fused carbocyclic and oxacyclic medium-sized rings: Competition between Grubbs-II catalyzed ring closing olefin metathesis and ring closing carbonyl-olefin metathesis
}

\author{
P CHAKRABORTY and S C ROY* \\ Department of Organic Chemistry, Indian Association for the Cultivation of Science, Jadavpur, \\ Kolkata 700 032, India \\ e-mail: ocscr@iacs.res.in
}

MS received 22 September 2016; accepted 23 October 2016

\begin{abstract}
A study towards diversity-oriented synthesis of optically active cyclopentane fused bicyclic frameworks has been accomplished. The common intermediate was prepared from commercially available starting material $(S)$-carvone. The observations on competition between Grubbs-II catalyzed ring closing metathesis (RCM) and ring closing carbonyl-olefin metathesis (RCCOM) were the key features of the study.
\end{abstract}

Keywords. Diversity-oriented synthesis; bicyclic frameworks; ring closing metathesis; Grubbs-II catalyst; carbonyl-olefin metathesis.

\section{Introduction}

The synchronous evolution of synthetic chemistry and biology-led clinical trials over the last decade have intensified the significance of small molecule synthesis. ${ }^{1}$ Small molecules are currently often treated as new drugs and drug candidates for their ability to unite to a pre-selected protein or other biological macromolecules in a specific manner with minimal chances of side effects. Thus, there is a constant switch over from cytotoxic chemotherapy to the consumption of small molecule cancer drugs that targets cancer cells at molecular level in a more efficient dose-dependent approach. ${ }^{2}$ Incessant research has been going on to create and enrich the library of small molecules by means of carbocycles with bicyclic motif of different ring sizes. ${ }^{3}$ Syntheses of these medium-sized bicyclic frameworks consequently have gained enormous attention as they form the structural core of a large number of biologically important natural products. ${ }^{4}$ When there are several reports for the synthetic approaches to five- and six-membered ring systems via common cyclization and cycloaddition reactions, seven- and eight-membered ring formations are not too much familiar due to considerable entropy loss in the transition state and large enthalpy loss due to trans-annular interactions. ${ }^{5}$

*For correspondence
Diversity-oriented synthetic strategies for designing such a category of biologically relevant bicyclic skeletons have been frequently exercised in active scientific discussions. Diversity-oriented synthesis (DOS) ${ }^{6}$ works with powerful planning algorithm which has numerous benefits over target-oriented synthesis (TOS) and has been successfully applied for constructing libraries of small molecules. In general, DOS is a protocol of synthesizing an array of compounds with structural diversity from a common synthetic intermediate. Though there are considerable amount of documentations of constructing nitrogen and oxygen containing heterocycles via DOS in the literature, ${ }^{7}$ synthesis of carbocycles with densely functionalized bicyclic moieties have been persued in limited ways. ${ }^{8}$ So, development of a practical and constructive diversity-oriented approach to improve the documentation of carbocycles with medium sized rings will cover synthetic as well as biological utility.

The literature survey of various natural products synthesis revealed that the core structures of a large number of naturally occurring biologically important molecules contains certain kind of bicyclic frameworks such as, for example, five-five, five-six, five-seven, or a five-eight fused bicyclic carbocycles (Scheme 1). ${ }^{9}$ Inclination towards the substituted cyclopentane-fused carbocycles in natural products attracted chemists for synthesis of such molecules for years.

Herein, we depict a diversity-oriented study on the synthesis of optically active, highly functionalized 
a) core structure with five-five fused bicyclic skeleton

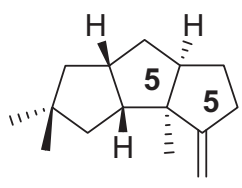

Hirsutene

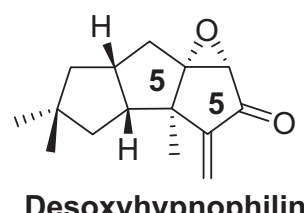

Desoxyhypnophilin

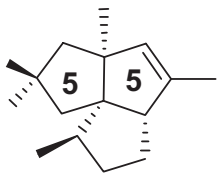

Pentalene

b) core structure with five-six fused bicyclic skeleton<smiles>CC1=C(CCO)C(=O)C2=C(C1)C(=O)C1C[C@]2(C)OC1=O</smiles>

(+)-Puraquinoic acid

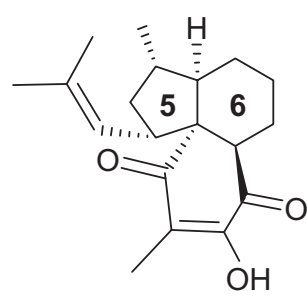

Elisabethin A<smiles>C=CC1C[C@H](Cc2ccc(OC)c(O)c2)[C@@H]2C(=O)C=CC(=C)[C@H]12</smiles>

Ottelione B

c) core structure with five-seven fused bicyclic skeleton<smiles>CC(C)=CCC[C@](C)(O)[C@H]1CC[C@@]2(C)CC=C(C)CC[C@]12C</smiles>

(-)-Tormesol

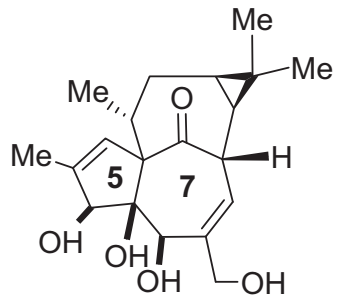

(+)-Ingenol

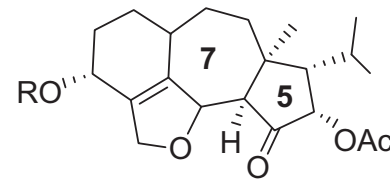

$\mathrm{R}=\mathrm{H}(-)$-Guanacastepene $\mathrm{E}$ $\mathbf{R}=$ Ac Heptemerone B

d) core structure with five-eight fused bicyclic skeleton

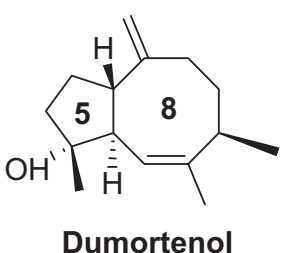

Dumortenol

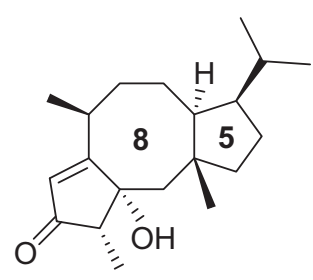

Anadensin

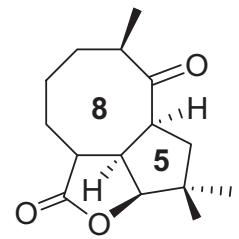

Asteriscanolide

Scheme 1. Natural products with different bicyclic framework as the core structure.

cyclopentane fused medium sized carbocyclic and oxacyclic frameworks using Grubbs-II catalyzed ring closing metathesis (RCM). ${ }^{10}$ A competition between RCM and ring closing carbonyl-olefin metathesis $(\mathrm{RCCOM})^{11}$ was observed depending on the size of the ring formation. The common intermediate for DOS was prepared from commercially available starting material $(S)$-carvone.

\section{Experimental}

The ${ }^{1} \mathrm{H}$ NMR spectra were recorded in $\mathrm{CDCl}_{3}$ on 300 , 400 and $500 \mathrm{MHz}$ and ${ }^{13} \mathrm{C}$ NMR spectra were recorded on 75,100 and $125 \mathrm{MHz}$ spectrometer, respectively, using TMS as an internal standard. High-resolution mass spectra (HRMS) were obtained using a Qtof Micro YA263 instrument. Ethyl acetate was dried over anhydrous calcium chloride. Petroleum ether of boiling range $60-80^{\circ} \mathrm{C}$ and diethyl ether were dried over sodium. Silica gel of 60-120 mesh was used for column chromatography. THF and toluene were super dried by distillation over sodium. DCM solvent was used after freshly distilled over $\mathrm{P}_{2} \mathrm{O}_{5}$. Except 2-iodoxybenzoic acid (IBX) oxidation and $30 \% \quad \mathrm{H}_{2} \mathrm{O}_{2}$ mediated peroxidation procedures, all the reactions were carried out either in argon or nitrogen atmosphere with oven-dried 
glass apparatus. A number of the compounds described are already reported in the literature and are characterized by NMR, IR and MASS spectral studies and compared them with authentic samples. ${ }^{12}$

\subsection{Preparation of $(R)-1-((1 S, 2 R, 3 S, 5 S)-3-(2,2-$ dimethyl-1,1-diphenylpropoxy)-2-methyl-5-(prop-1- en-2-yl)cyclopentyl)prop-2-en-1-ol (9a)}

$2.44 \mathrm{~mL}$ of $1.0 \mathrm{M}$ solution of vinyl magnesium bromide ( $2.44 \mathrm{mmol})$ in THF was added dropwise to a solution of the aldehyde $\mathbf{8}^{12}$ (500 mg, $1.22 \mathrm{mmol}$ ) in THF $(15 \mathrm{~mL})$ at $-78^{\circ} \mathrm{C}$ under Argon atmosphere. Stirring was continued for $3 \mathrm{~h}$ at that temperature. The reaction was monitored by checking TLC. It was then quenched with saturated aqueous ammonium chloride solution $(2 \mathrm{~mL})$. THF was removed under reduced pressure and the residue was extracted with diethyl ether $(3 \times$ $20 \mathrm{~mL}$ ). The combined organic extract was washed with brine and finally dried over anhydrous sodium sulfate. The solvent was removed under reduced pressure and the residue obtained was purified by column chromatography over silica gel (3\% ethyl acetate in light petroleum) to furnish 9a $(430 \mathrm{mg}, 81 \%)$ as a viscous liquid. ${ }^{1} \mathrm{H}$ NMR $\left(500 \mathrm{MHz}, \mathrm{CDCl}_{3}\right): \delta 1.00(\mathrm{~d}, J=7.5 \mathrm{~Hz}, 3 \mathrm{H})$, $1.08(\mathrm{~s}, 9 \mathrm{H}), 1.52(\mathrm{q}, J=6.0 \mathrm{~Hz}, 1 \mathrm{H}), 1.70-1.77$ (m, 1H), 1.89 (s, 3H), 1.93-1.97 (m, 1H), 2.17-2.21 (m, 1H), 3.11-3.17 (m, 1H), 4.29-4.33 (m, 2H), 4.72 $(\mathrm{s}, 1 \mathrm{H}), 4.89(\mathrm{~s}, 1 \mathrm{H}), 5.05$ (doublet with further splitting, $J=9.0 \mathrm{~Hz}, 1 \mathrm{H}$ ), 5.19 (doublet with further splitting, $J=13.5 \mathrm{~Hz}, 1 \mathrm{H}), 5.75-5.82(\mathrm{~m}, 1 \mathrm{H}), 7.33-$ $7.42(\mathrm{~m}, 6 \mathrm{H}), 7.64-7.68(\mathrm{~m}, 4 \mathrm{H}) ;{ }^{13} \mathrm{C}$ NMR $(125 \mathrm{MHz}$, $\left.\mathrm{CDCl}_{3}\right): \delta 17.1,19.6,24.5,27.2,27.3,37.9,39.6,45.8$, 51.1, 71.8, 76.4, 110.1, 113.3, 127.5, 129.5, 134.4, 135.0, 135.9, 136.0, 136.1, 139.8, 148.1; HRMS: calcd for $\mathrm{C}_{28} \mathrm{H}_{38} \mathrm{O}_{2} \mathrm{Si}[\mathrm{M}+\mathrm{Na}]^{+}$457.2539; found: 457.2541.

\subsection{Preparation of $(R)-1-((1 S, 2 R, 3 S, 5 S)-3-(2,2-$ dimethyl-1,1-diphenylpropoxy)-2-methyl-5-(prop-1- en-2-yl)cyclopentyl)but-3-en-1-ol (9b)}

Compound 9b (77\%) was prepared from 8 and freshly prepared allyl Grignard reagent, using the same procedure used for synthesizing 9a. ${ }^{1} \mathrm{H}$ NMR $(500 \mathrm{MHz}$, $\left.\mathrm{CDCl}_{3}\right): \delta 1.01-1.11(\mathrm{~m}, 12 \mathrm{H}), 1.48-1.55(\mathrm{~m}, 1 \mathrm{H})$, $1.64-1.74(\mathrm{~m}, 1 \mathrm{H}), 1.83(\mathrm{~s}, 3 \mathrm{H}), 1.88(\mathrm{t}, J=7.0 \mathrm{~Hz}$, $1 \mathrm{H}), 2.07-2.19(\mathrm{~m}, 2 \mathrm{H}), 2.28(\mathrm{q}, J=6.0 \mathrm{~Hz}, 1 \mathrm{H})$, $3.08-3.10(\mathrm{~m}, 1 \mathrm{H}), 3.70(\mathrm{~m}, 1 \mathrm{H}), 4.34(\mathrm{t}, J=4.0 \mathrm{~Hz}$, $1 \mathrm{H}), 4.67(\mathrm{~s}, 1 \mathrm{H}), 4.84(\mathrm{~s}, 1 \mathrm{H}), 5.04-5.08(\mathrm{~m}, 2 \mathrm{H})$, 5.76-5.81 (m, 1H), 7.25-7.43 (m, 6H), 7.63-7.70 $(\mathrm{m}, 4 \mathrm{H}) ;{ }^{13} \mathrm{C}$ NMR $\left(75 \mathrm{MHz}, \mathrm{CDCl}_{3}\right): \delta 17.2,19.6$, 24.5, 27.3, 38.0, 39.6, 39.8, 46.2, 50.5, 71.4, 76.5, $110.0,117.1,127.5,127.6,129.5,134.5,135.0,136.0$,
136.1, 148.0; HRMS: calcd for $\mathrm{C}_{29} \mathrm{H}_{40} \mathrm{O}_{2} \mathrm{Si}[\mathrm{M}+\mathrm{Na}]^{+}$ 471.2695; found: 471.2695 .

\subsection{Preparation of $(R)-1-((1 S, 2 R, 3 S, 5 S)-3-(2,2-$}

dimethyl-1,1-diphenylpropoxy)-2-methyl-5-(prop-1en-2-yl)cyclopentyl)pent-4-en-1-ol (9c)

Compound 9c (67\%) was prepared from 8 and freshly prepared homoallyl Grignard reagent, using the same procedure used for synthesizing 9a. ${ }^{1} \mathrm{H}$ NMR $\left(500 \mathrm{MHz}, \mathrm{CDCl}_{3}\right): \delta 1.06-1.09(\mathrm{~m}, 12 \mathrm{H}), 1.36-1.42$ (m, 1H), 1.50-1.73 (m, 4H), $1.86(\mathrm{~s}, 3 \mathrm{H}), 2.03-2.07$ (m, 1H), 2.16-2.20 (m, 1H), 2.25-2.29 (m, 1H), 3.10$3.15(\mathrm{~m}, 1 \mathrm{H}), 3.70-3.71(\mathrm{~m}, 1 \mathrm{H}), 4.34-4.35(\mathrm{~m}, 1 \mathrm{H})$, $4.69(\mathrm{~s}, 1 \mathrm{H}), 4.86(\mathrm{~s}, 1 \mathrm{H}), 4.94-5.04(\mathrm{~m}, 2 \mathrm{H}), 5.79-$ $5.84(\mathrm{~m}, 1 \mathrm{H}), 7.34-7.44(\mathrm{~m}, 6 \mathrm{H}), 7.65-7.69(\mathrm{~m}, 4 \mathrm{H})$; ${ }^{13} \mathrm{C} \mathrm{NMR}\left(125 \mathrm{MHz}, \mathrm{CDCl}_{3}\right): \delta 17.3,19.7,24.6,27.2$, 27.3, 27.6, 30.8, 34.1, 38.0, 39.6, 46.2, 50.6, 71.3, 76.7, 109.7, 114.6, 127.5, 127.6, 127.9, 129.5, 129.6, 134.5, 135.0, 135.8, 136.0, 136.1, 138.8, 148.6; HRMS: calcd for $\mathrm{C}_{30} \mathrm{H}_{42} \mathrm{O}_{2} \mathrm{Si}[\mathrm{M}+\mathrm{Na}]^{+}$485.2852; found: 485.2853.

\subsection{Preparation of $(R)-1-((1 S, 2 R, 3 S, 5 S)-3-(2,2-$}

dimethyl-1,1-diphenylpropoxy)-2-methyl-5-(prop-1en-2-yl)cyclopentyl)hex-5-en-1-ol (9d)

Compound 9d (64\%) was prepared from 8 and freshly prepared corresponding Grignard reagent, using the same procedure used for synthesizing 9a. ${ }^{1} \mathrm{H}$ NMR $\left(500 \mathrm{MHz}, \mathrm{CDCl}_{3}\right): \delta 1.02-1.08(\mathrm{~m}, 12 \mathrm{H}), 1.25-1.68$ $(\mathrm{m}, 7 \mathrm{H}), 1.85(\mathrm{~s}, 3 \mathrm{H}), 2.02-2.05(\mathrm{~m}, 2 \mathrm{H}), 2.23-2.25$ $(\mathrm{m}, 1 \mathrm{H}), 3.10(\mathrm{~m}, 1 \mathrm{H}), 3.66(\mathrm{~m}, 1 \mathrm{H}), 4.31-4.32(\mathrm{~m}$, $1 \mathrm{H}), 4.68(\mathrm{~s}, 1 \mathrm{H}), 4.85(\mathrm{~s}, 1 \mathrm{H}), 4.92-5.00(\mathrm{~m}, 1 \mathrm{H})$, 5.76-5.80 (m, 1H), 7.33-7.41 (m, 6H), 7.62-7.68 (m, $4 \mathrm{H}) ;{ }^{13} \mathrm{C} \mathrm{NMR}\left(125 \mathrm{MHz}, \mathrm{CDCl}_{3}\right): \delta 17.3,19.7,24.6$, 25.9, 27.1, 27.2, 27.3, 33.8, 34.4, 38.0, 39.6, 46.2, 50.6, $71.8,76.7,109.7,110.1,114.6,127.5,127.6,127.8$, 129.6, 129.8, 130.0, 134.5, 135.1, 136.0, 136.1, 138.9, 139.0, 148.6; HRMS: calcd for $\mathrm{C}_{31} \mathrm{H}_{44} \mathrm{O}_{2} \mathrm{Si}[\mathrm{M}+\mathrm{Na}]^{+}$ 499.3008; found: 499.3009 .

\subsection{Preparation of $(R)-1-((1 S, 2 R, 3 S, 5 S)-3-(2,2-$} dimethyl-1,1-diphenylpropoxy)-2-methyl-5-(prop-1en-2-yl)cyclopentyl)hept-6-en-1-ol (9e)

Compound 9e (63\%) was prepared from 8 and freshly prepared corresponding Grignard reagent, using the same procedure used for synthesizing 9a. ${ }^{1} \mathrm{H}$ NMR $\left(500 \mathrm{MHz}, \mathrm{CDCl}_{3}\right): \delta 1.04-1.12(\mathrm{~m}, 12 \mathrm{H}), 1.26-1.72$ (m, 9H), 1.85 (s, 3H), 2.02-2.09 (m, 2H), 2.23-2.27 $(\mathrm{m}, 1 \mathrm{H}), 3.11(\mathrm{~m}, 1 \mathrm{H}), 3.66(\mathrm{~m}, 1 \mathrm{H}), 4.32(\mathrm{t}, J=$ $4.0 \mathrm{~Hz}, 1 \mathrm{H}), 4.68(\mathrm{~s}, 1 \mathrm{H}), 4.85(\mathrm{~s}, 1 \mathrm{H}), 4.93(\mathrm{dd}$, $J=1.0,10.5 \mathrm{~Hz}, 1 \mathrm{H}), 4.99(\mathrm{dd}, J=1.5,17.0 \mathrm{~Hz}$, 
1H), 5.77-5.83 (m, 1H), 7.33-7.43 (m, 6H), 7.63-7.70 $(\mathrm{m}, 4 \mathrm{H}) ;{ }^{13} \mathrm{C} \mathrm{NMR}\left(125 \mathrm{MHz}, \mathrm{CDCl}_{3}\right): \delta 17.3,20.4$, $24.6,26.2,27.1,27.2,27.3,29.1,33.9,34.9,38.0$, $39.6,46.1,50.6,71.99,109.7,114.4,127.5,127.7$, $127.8,129.5,129.8,129.9,134.5,135.1,136.0,136.1$, 139.1, 148.6; HRMS: calcd for $\mathrm{C}_{32} \mathrm{H}_{46} \mathrm{O}_{2} \mathrm{Si}[\mathrm{M}+\mathrm{Na}]^{+}$ 513.3165; found: 513.3169 .

2.6 Preparation of 1-((1S,2R,3S,5S)-3-(2,2-dimethyl1,1-diphenylpropoxy)-2-methyl-5-(prop-1-en-2-yl) cyclopentyl)prop-2-en-1-one (10a)

Solid Dess-Martin periodinate $(730 \mathrm{mg}, 1.72 \mathrm{mmol}$ ) and $\mathrm{NaHCO}_{3}(150 \mathrm{mg}, 1.8 \mathrm{mmol})$ were added to a stirring solution of alcohol 9a $(300 \mathrm{mg}, 0.70 \mathrm{mmol})$ dissolved in $20 \mathrm{~mL}$ of DCM at room temperature and the reaction was continued for $3 \mathrm{~h}$. The completion of the reaction was monitored by TLC before it was quenched by a mixture of saturated aqueous solution of $\mathrm{Na}_{2} \mathrm{~S}_{2} \mathrm{O}_{3}$ and $\mathrm{NaHCO}_{3}$. The mixture was then extracted with diethyl ether $(3 \times 20 \mathrm{~mL})$. The combined organic extract was washed with brine and finally dried over anhydrous sodium sulfate. The solvent was removed under reduced pressure and the residue obtained was purified by column chromatography over silica gel $(3 \%$ ethyl acetate in light petroleum) to furnish 10a $(280 \mathrm{mg}$, $92 \%)$ as a viscous liquid. $[\alpha]_{\mathrm{D}}^{25}=26.07(\mathrm{c}=2.03$, $\left.\mathrm{CHCl}_{3}\right) ;{ }^{1} \mathrm{H} \mathrm{NMR}\left(500 \mathrm{MHz}, \mathrm{CDCl}_{3}\right): \delta 1.01(\mathrm{~d}, J=$ $6.5 \mathrm{~Hz}, 3 \mathrm{H}), 1.11(\mathrm{~s}, 9 \mathrm{H}), 1.51(\mathrm{~s}, 3 \mathrm{H}), 1.72-1.73(\mathrm{~m}$. $2 \mathrm{H}), 2.40(\mathrm{t}, J=3.5 \mathrm{~Hz}, 1 \mathrm{H}), 3.35-3.37(\mathrm{~m}, 2 \mathrm{H})$, 4.36-4.37 (m, 1H), $4.63(\mathrm{~s}, 1 \mathrm{H}), 4.68(\mathrm{~s}, 1 \mathrm{H}), 5.71(\mathrm{~d}$, $J=11 \mathrm{~Hz}, 1 \mathrm{H}), 6.18(\mathrm{~d}, J=17.5 \mathrm{~Hz}, 1 \mathrm{H}), 6.36-$ $6.42(\mathrm{~m}, 1 \mathrm{H}), 7.37-7.45(\mathrm{~m}, 6 \mathrm{H}), 7.68-7.69(\mathrm{~m}, 4 \mathrm{H})$; ${ }^{13} \mathrm{C}$ NMR (125 MHz, $\left.\mathrm{CDCl}_{3}\right): \delta 14.5,19.6,21.3,27.2$, 40.2, 42.8, 47.7, 57.4, 76.9, 113.1, 127.0, 127.6, 129.7, 134.1, 134.8, 135.9, 136.0, 137.6, 145.3, 201.9; HRMS: calcd for $\mathrm{C}_{28} \mathrm{H}_{36} \mathrm{O}_{2} \mathrm{Si}[\mathrm{M}+\mathrm{H}]^{+}$433.2557; found: 433.2558 .

2.7 Preparation of 1-((1S,2R,3S,5S)-3-(2,2-dimethyl1,1-diphenylpropoxy)-2-methyl-5-(prop-1-en-2-yl) cyclopentyl)but-3-en-1-one (10b)

Compound $\mathbf{1 0 b}(90 \%)$ was prepared from $\mathbf{9 b}$ using the same procedure used for synthesizing 10a. $[\alpha]_{\mathrm{D}}^{25}=$ $27.78\left(\mathrm{c}=0.977, \mathrm{CHCl}_{3}\right) ;{ }^{1} \mathrm{H} \mathrm{NMR}(500 \mathrm{MHz}$, $\left.\mathrm{CDCl}_{3}\right): \delta 0.95(\mathrm{~d}, J=6.5 \mathrm{~Hz}, 3 \mathrm{H}), 1.09(\mathrm{~s}, 9 \mathrm{H}), 1.53$ (s, 3H), 1.66-1.70 (m, 2H), 2.32-2.37 (m, 1H), 3.08$3.16(\mathrm{~m}, 2 \mathrm{H}), 3.22-3.27(\mathrm{~m}, 1 \mathrm{H}), 3.32-3.38(\mathrm{~m}, 1 \mathrm{H})$, 4.31 (appeared as singlet, $1 \mathrm{H}$ ), 4.71-4.72 (overlapping of two singlets of olefinic protons, $2 \mathrm{H}), 5.09$ (d, $J=$ $17 \mathrm{~Hz}, 1 \mathrm{H}), 5.15(\mathrm{~d}, J=10.5 \mathrm{~Hz}, 1 \mathrm{H}), 5.86-5.91(\mathrm{~m}$, $1 \mathrm{H}), 7.35-7.44(\mathrm{~m}, 6 \mathrm{H}), 7.65(\mathrm{~d}, J=7.5 \mathrm{~Hz}, 4 \mathrm{H})$;
${ }^{13} \mathrm{C}$ NMR (75 MHz, $\left.\mathrm{CDCl}_{3}\right): \delta 14.4,19.6,20.8,27.2$, 27.6, 40.0, 42.2, 47.2, 49.0, 60.1, 113.4, 118.4, 127.6, $127.9,129.7,131.1,134.1,134.9,135.8,135.9,136.0$, 145.8, 210.0; HRMS: calcd for $\mathrm{C}_{29} \mathrm{H}_{38} \mathrm{O}_{2} \mathrm{Si}[\mathrm{M}+\mathrm{H}]^{+}$ 447.2714; found: 447.2711.

2.8 Preparation of 1-((1S,2R,3S,5S)-3-(2,2-dimethyl1,1-diphenylpropoxy)-2-methyl-5-(prop-1-en-2-yl) cyclopentyl)pent-4-en-1-one (10c)

Compound 10c (89\%) was prepared from 9c using the same procedure used for synthesizing 10a. $[\alpha]_{\mathrm{D}}^{25}=$ $25.64\left(\mathrm{c}=3.10, \mathrm{CHCl}_{3}\right) ;{ }^{1} \mathrm{H} \mathrm{NMR}\left(500 \mathrm{MHz}, \mathrm{CDCl}_{3}\right)$ : $\delta 0.94(\mathrm{~d}, J=6.5 \mathrm{~Hz}, 3 \mathrm{H}), 1.09(\mathrm{~s}, 9 \mathrm{H}), 1.52-1.67$ (m, 5H), 2.22-2.43 (m, 4H), 2.54-2.60 (m, 1H), 3.06$3.10(\mathrm{~m}, 1 \mathrm{H}), 3.34(\mathrm{t}, J=8.0 \mathrm{~Hz}, 1 \mathrm{H}), 4.30$ (appeared as singlet, $1 \mathrm{H}$ ), 4.67-4.70 (overlapping of two singlets of olefinic protons, $2 \mathrm{H}), 4.93-5.02(\mathrm{~m}, 2 \mathrm{H}), 5.77-5.80$ $(\mathrm{m}, 1 \mathrm{H}), 7.36-7.41(\mathrm{~m}, 6 \mathrm{H}), 7.65$ (appeared as singlet, $4 \mathrm{H}) ;{ }^{13} \mathrm{C} \mathrm{NMR}\left(125 \mathrm{MHz}, \mathrm{CDCl}_{3}\right): \delta 14.5,19.6$, $21.0,27.0,27.2,27.8,39.9,42.3,43.6,47.2,60.5$, 113.2, 115.0, 127.6, 129.7, 134.2, 134.9, 136.0, 137.6, 145.8, 211.4; HRMS: calcd for $\mathrm{C}_{30} \mathrm{H}_{40} \mathrm{O}_{2} \mathrm{Si}[\mathrm{M}+\mathrm{Na}]^{+}$ 483.2695; found: 483.2694 .

\subsection{Preparation of $1-((1 S, 2 R, 3 S, 5 S)-3-(2,2$-dimethyl-}

1,1-diphenylpropoxy)-2-methyl-5-(prop-1-en-2-yl) cyclopentyl)hex-5-en-1-one (10d)

Compound 10d (88\%) was prepared from 9d using the same procedure used for synthesizing 10a. $[\alpha]_{\mathrm{D}}^{25}=$ $25.87\left(\mathrm{c}=2.43, \mathrm{CHCl}_{3}\right) ;{ }^{1} \mathrm{H} \mathrm{NMR}\left(500 \mathrm{MHz}, \mathrm{CDCl}_{3}\right)$ : $\delta 0.95(\mathrm{~d}, J=7.0 \mathrm{~Hz}, 3 \mathrm{H}), 1.08(\mathrm{~s}, 9 \mathrm{H}), 1.50$ $1.71(\mathrm{~m}, 7 \mathrm{H}), 2.00-2.04(\mathrm{~m}, 2 \mathrm{H}), 2.28-2.35(\mathrm{~m}, 2 \mathrm{H})$, $2.45-2.52(\mathrm{~m}, 1 \mathrm{H}), 3.07(\mathrm{t}, J=10.0 \mathrm{~Hz}, 1 \mathrm{H}), 3.32$ $(\mathrm{q}, J=9.0 \mathrm{~Hz}, 1 \mathrm{H}), 4.30-4.31(\mathrm{~m}, 1 \mathrm{H}), 4.67(\mathrm{~s}$, 1H), 4. .69 (s, 1H), 4.95-5.03 (m, 2H), 5.72-5.79 (m, 1H), 7.35-7.43 (m, 6H), 7.64-7.66 (m, 4H); ${ }^{13} \mathrm{C} \mathrm{NMR}$ $\left(125 \mathrm{MHz}, \mathrm{CDCl}_{3}\right): \delta 14.2,14.5,19.6,21.0,22.7,27.2$, $29.8,31.7,33.2,39.9,42.3,43.7,47.2,60.5,113.7$, 115.0, 127.6, 129.7, 134.2, 134.9, 135.9, 136.0, 138.4, 145.9, 212.1; HRMS: calcd for $\mathrm{C}_{31} \mathrm{H}_{42} \mathrm{O}_{2} \mathrm{Si}[\mathrm{M}+\mathrm{Na}]^{+}$ 497.2852; found: 497.2854 .

2.10 Preparation of 1-((1S,2R,3S,5S)-3-(2,2-dimethyl-1, 1-diphenylpropoxy)-2-methyl-5-(prop-1-en-2-yl) cyclopentyl)hept-6-en-1-one (10e)

Compound 10e (89\%) was prepared from 9e using the same procedure used for synthesizing 10a. $[\alpha]_{\mathrm{D}}^{25}=$ $27.52\left(\mathrm{c}=2.05, \mathrm{CHCl}_{3}\right) ;{ }^{1} \mathrm{H} \mathrm{NMR}\left(500 \mathrm{MHz}, \mathrm{CDCl}_{3}\right)$ : $\delta 0.93-1.68(\mathrm{~m}, 21 \mathrm{H}), 2.03(\mathrm{q}, J=7.5 \mathrm{~Hz}, 2 \mathrm{H})$, 2.29-2.34 (m, 2H), 2.44-2.48 (m, 1H), $3.07(\mathrm{t}, J=$ 
$10.0 \mathrm{~Hz}, 1 \mathrm{H}), 3.32(\mathrm{q}, J=10.0 \mathrm{~Hz}, 1 \mathrm{H}), 4.30(\mathrm{~m}, 1 \mathrm{H})$, 4.67-4.69 (overlapping of two singlets of olefinic protons, $2 \mathrm{H}), 4.92-5.00(\mathrm{~m}, 2 \mathrm{H}), 5.74-5.82(\mathrm{~m}, 1 \mathrm{H}), 7.34$ 7.43 (m, 6H), 7.63-7.65 (m, 4H); ${ }^{13} \mathrm{C}$ NMR $(125 \mathrm{MHz}$, $\left.\mathrm{CDCl}_{3}\right): \delta 14.2,14.5,19.6,21.0,22.7,23.1,25.4,27.0$, 27.2, 28.6, 29.8, 31.7, 33.7, 39.9, 42.3, 44.3, 47.2, 60.4, 113.1, 114.6, 127.6, 129.7, 134.2, 134.9, 136.0, 138.8, 145.9, 212.2; HRMS: calcd for $\mathrm{C}_{32} \mathrm{H}_{44} \mathrm{O}_{2} \mathrm{Si}[\mathrm{M}+\mathrm{Na}]^{+}$ 511.3008; found: 511.3007 .

2.11 Preparation of (3aS,5S,6R,6aS)-5-(2,2-dimethyl1,1-diphenylpropoxy)-4,5,6,6a-tetrahydro-3,6-dimethylpentalen-1(3aH)-one (11a)

To a stirred solution of $\mathbf{1 0 a}(100 \mathrm{mg}, 0.23 \mathrm{mmol})$ in dry toluene at room temperature under argon atmosphere was added Grubbs second generation catalyst $(20 \mathrm{mg}$, $10 \mathrm{~mol} \%)$. The reaction mixture was then refluxed for $3 \mathrm{~h}$ and the reaction was monitored by TLC. The solvent was concentrated under vacuum and the resulting residue was purified by column chromatography over silica gel (10\% ethyl acetate in light petroleum) to furnish the cyclized product $11 \mathrm{a}(86 \mathrm{mg}, 92 \%) .{ }^{1} \mathrm{H}$ $\operatorname{NMR}\left(500 \mathrm{MHz}, \mathrm{CDCl}_{3}\right):[\alpha]_{\mathrm{D}}^{25}=-17.18(\mathrm{c}=4.08$, $\left.\mathrm{CHCl}_{3}\right) ; \delta 1.07$ (s, 9H), $1.18(\mathrm{~d}, J=6.5 \mathrm{~Hz}, 3 \mathrm{H}), 1.26-$ $1.31(\mathrm{~m}, 1 \mathrm{H}), 1.81-1.85(\mathrm{~m}, 1 \mathrm{H}), 1.88(\mathrm{~s}, 3 \mathrm{H}), 2.00(\mathrm{q}$, $J=6.0 \mathrm{~Hz}, 1 \mathrm{H}), 2.58(\mathrm{t}, J=6.0 \mathrm{~Hz}, 1 \mathrm{H}), 3.19(\mathrm{dd}$, $J=15,6.5 \mathrm{~Hz}, 1 \mathrm{H}), 4.10(\mathrm{q}, J=5.0 \mathrm{~Hz}, 1 \mathrm{H}), 5.66$ (s, 1H), 7.35-7.38 (m, 4H), 7.41-7.43 (m, 2H), 7.62 $(\mathrm{m}, 4 \mathrm{H}) ;{ }^{13} \mathrm{C}$ NMR $\left(125 \mathrm{MHz}, \mathrm{CDCl}_{3}\right): \delta 14.5,17.6$, 19.5, 27.1, 35.2, 41.7, 47.7, 56.7, 76.9, 127.6, 127.7, $129.2,129.8,133.8,134.6,135.8,180.2$; HRMS: calcd for $\mathrm{C}_{26} \mathrm{H}_{32} \mathrm{O}_{2} \mathrm{Si}[\mathrm{M}+\mathrm{Na}]^{+}$427.2069; found: 427.2068.

2.12 Preparation of (2S,3R,3aS,7aS)-2-(2,2-dimethyl1,1-diphenylpropoxy)-1,2,3,3a-tetrahydro-3,7-dimethyl$5 H$-inden-4(7aH)-one (11b)

Compound 11b (93\%) was prepared from 10b using the same procedure used for synthesizing 11a. $[\alpha]_{\mathrm{D}}^{25}=$ -5.94 (c = 2.20, $\left.\mathrm{CHCl}_{3}\right) ;{ }^{1} \mathrm{H} \mathrm{NMR}\left(400 \mathrm{MHz}, \mathrm{CDCl}_{3}\right)$ : $\delta 1.08$ (appeared as singlet, $12 \mathrm{H}), 1.24-1.33(\mathrm{~m}, 1 \mathrm{H})$, $1.54(\mathrm{~s}, 3 \mathrm{H}), 1.91$ (dd, $J=8.0,12.8 \mathrm{~Hz}, 1 \mathrm{H}), 2.16-$ $2.21(\mathrm{~m}, 1 \mathrm{H}), 2.67(\mathrm{t}, J=9.6 \mathrm{~Hz}, 1 \mathrm{H}), 2.75-2.89(\mathrm{~m}$, $2 \mathrm{H}), 3.17$ (q, $J=8.8 \mathrm{~Hz}, 1 \mathrm{H}), 4.23$ (appeared as singlet, $1 \mathrm{H}), 5.22$ (appeared as singlet, $1 \mathrm{H}), 7.38-7.44(\mathrm{~m}$, 6H), 7.66-7.67 (m, 4H); $\left.{ }^{13} \mathrm{C} \mathrm{NMR} \mathrm{(100} \mathrm{MHz,} \mathrm{CDCl}_{3}\right)$ : $\delta 14.3,19.6,21.4,27.2,38.2,41.4,43.5,45.2,55.3$, 116.0, 127.6, 127.7, 129.7, 133.9, 134.9, 135.9, 136.0, 137.6, 212.0; HRMS: calcd for $\mathrm{C}_{27} \mathrm{H}_{34} \mathrm{O}_{2} \mathrm{Si}[\mathrm{M}+\mathrm{H}]^{+}$ 441.2226; found: 441.2228 .
2.13 Preparation of $(Z, 2 S, 3 R, 3 a S, 8 a S)-2-(2,2$-dimethyl1,1-diphenylpropoxy)-1,2,3,3a,5,6-hexahydro-3,8dimethylazulen-4(8aH)-one (11c)

Compound 11c (89\%) was prepared from 10c using the same procedure used for synthesizing 11a. $[\alpha]_{\mathrm{D}}^{25}=$ $26.96\left(\mathrm{c}=1.49, \mathrm{CHCl}_{3}\right) ;{ }^{1} \mathrm{H}$ NMR $\left(500 \mathrm{MHz}, \mathrm{CDCl}_{3}\right)$ : $\delta 0.96(\mathrm{~d}, J=7.0 \mathrm{~Hz}, 3 \mathrm{H}), 1.09(\mathrm{~s}, 9 \mathrm{H}), 1.25-1.42$ $(\mathrm{m}, 2 \mathrm{H}), 1.49(\mathrm{~s}, 3 \mathrm{H}), 1.71(\mathrm{dd}, J=7.0,13.0 \mathrm{~Hz}$, $1 \mathrm{H}), 2.14-2.17(\mathrm{~m}, 1 \mathrm{H}), 2.42-2.54(\mathrm{~m}, 3 \mathrm{H}), 3.14$ $(\mathrm{dd}, J=9.0,11.5 \mathrm{~Hz}, 1 \mathrm{H}), 3.46-3.52(\mathrm{~m}, 1 \mathrm{H})$, 4.26 (t, $J=3.5 \mathrm{~Hz}, 1 \mathrm{H}), 5.53$ (appeared as singlet, $1 \mathrm{H}$ ), 7.35-7.44 (m, 6H), 7.66-7.68 (m, 4H); ${ }^{13} \mathrm{C}$ NMR $\left(125 \mathrm{MHz}, \mathrm{CDCl}_{3}\right): \delta 15.0,19.6,22.1,23.5,27.3,39.6$, 40.5, 42.9, 44.7, 58.8, 76.2, 124.0, 127.6, 127.7, 129.7, 135.0, 136.0, 136.1, 140.5, 211.5; HRMS: calcd for $\mathrm{C}_{28} \mathrm{H}_{36} \mathrm{O}_{2} \mathrm{Si}[\mathrm{M}+\mathrm{Na}]^{+}$455.2382; found: 455.2385.

2.14 Preparation of (Z,2S,3R,3aS,9aS)-2-(2,2-dimethyl-1, 1-diphenylpropoxy)-1,2,3,3a,6,7-hexahydro-3,9-dimethyl$5 H$-cyclopenta[8]annulen-4(9aH)-one (11d) and 1-((1R, 2S,3R,5S)-2-methyl-3-(2,2,3,3-tetramethylbutyl)-5-(prop1-en-2-yl)cyclopentyl)cyclopent-1-ene (11e)

To a stirred solution of $\mathbf{1 0 d}(200 \mathrm{mg}, 0.42 \mathrm{mmol})$ in dry toluene at room temperature under argon atmosphere was added Grubbs second generation catalyst $(36 \mathrm{mg}$, $10 \mathrm{~mol} \%)$. The reaction mixture was then refluxed for $3 \mathrm{~h}$ and the reaction was monitored by TLC. The solvent was concentrated under vacuum. The resulting residue containing the mixture of $\mathbf{1 1 d}$ and 11e was separated by column chromatography over silica gel (5\% ethyl acetate in light petroleum) to furnish 11d (56 mg, 30\%) and 11e $(13 \mathrm{mg}, 7 \%)$. Spectral data of 11d: $[\alpha]_{\mathrm{D}}^{25}=$ $23.08\left(\mathrm{c}=0.77, \mathrm{CHCl}_{3}\right) ;{ }^{1} \mathrm{H} \mathrm{NMR}\left(500 \mathrm{MHz}, \mathrm{CDCl}_{3}\right)$ : $\delta 0.95(\mathrm{~d}, J=5.0 \mathrm{~Hz}, 3 \mathrm{H}), 1.09(\mathrm{~s}, 9 \mathrm{H}), 1.50$ $1.72(\mathrm{~m}, 7 \mathrm{H}), 1.83-1.89(\mathrm{~m}, 1 \mathrm{H}), 2.02-2.08(\mathrm{~m}, 1 \mathrm{H})$, 2.26-2.32 (m, 2H), 2.55-2.61 (m, 1H), 3.31-3.36 (m, $1 \mathrm{H}), 3.73-3.77(\mathrm{~m}, 1 \mathrm{H}), 4.37-4.44(\mathrm{~m}, 1 \mathrm{H}), 5.32(\mathrm{t}$, $J=10.0 \mathrm{~Hz}, 1 \mathrm{H}), 7.36-7.44(\mathrm{~m}, 6 \mathrm{H}), 7.65-7.68(\mathrm{~m}$, $4 \mathrm{H}) ;{ }^{13} \mathrm{C}$ NMR $\left(125 \mathrm{MHz}, \mathrm{CDCl}_{3}\right): \delta 14.2,14.3,19.6$, 20.8, 22.1, 22.8, 22.9, 23.8, 25.4, 27.0, 27.2, 27.4, 29.1, 29.2, 29.5, 29.6, 29.8, 30.3, 31.7, 32.0, 33.9, 34.6, 34.8, 38.5, 39.1, 39.7, 40.4, 44.4, 58.9, 65.9, 126.6, 127.6, 127.7, 129.7, 136.0, 136.0, 136.1, 213.2; HRMS: calcd for $\mathrm{C}_{29} \mathrm{H}_{38} \mathrm{O}_{2} \mathrm{Si}[\mathrm{M}+\mathrm{Na}]^{+}$469.2539; found: 469.2537 . Spectral data of 11e: $[\alpha]_{\mathrm{D}}^{25}=19.00\left(\mathrm{c}=0.71, \mathrm{CHCl}_{3}\right)$; ${ }^{1} \mathrm{H}$ NMR $\left(500 \mathrm{MHz}, \mathrm{CDCl}_{3}\right): \delta 0.94(\mathrm{t}, J=5.0 \mathrm{~Hz}$, $3 \mathrm{H}), 1.09(\mathrm{~s}, 9 \mathrm{H}), 1.52-1.71(\mathrm{~m}, 7 \mathrm{H}), 1.89-1.99(\mathrm{~m}$, $2 \mathrm{H}), 2.25-2.37(\mathrm{~m}, 2 \mathrm{H}), 2.43-2.51(\mathrm{~m}, 1 \mathrm{H}), 3.07$ (t, $J=10.0 \mathrm{~Hz}, 1 \mathrm{H}), 3.30-3.35(\mathrm{~m}, 1 \mathrm{H}), 4.31$ (appeared as singlet, $1 \mathrm{H}), 4.67(\mathrm{~S}, 1 \mathrm{H}), 4.69(\mathrm{~s}, 1 \mathrm{H}), 5.34-5.38(\mathrm{~m}$, 1H), 7.35-7.44 (m, 6H), 7.64-7.66 (m, 4H); ${ }^{13} \mathrm{C} \mathrm{NMR}$ 
$\left(100 \mathrm{MHz}, \mathrm{CDCl}_{3}\right): \delta 14.6,19.6,21.1,23.5,26.8,27.3$, 32.1, 40.0, 42.3, 43.8, 47.2, 60.5, 113.1, 127.7, 129.7, 130.4, 134.2, 134.9, 136.0, 136.1, 145.9; HRMS: calcd for $\mathrm{C}_{30} \mathrm{H}_{40} \mathrm{OSi}[\mathrm{M}+\mathrm{Na}]^{+}$467.2746; found: 467.2747.

2.15 Preparation of $1-((1 S, 2 R, 3 S, 4 S)-3$-cyclohexenyl2-methyl-4-(prop-1-en-2-yl)cyclopentyloxy)-2,2-dimethyl1,1-diphenylpropane (11f)

Compound 11f 10\%) was prepared from 10e using the same procedure used for synthesizing 11a. $[\alpha]_{\mathrm{D}}^{25}=$ $15.85\left(\mathrm{c}=0.955, \mathrm{CHCl}_{3}\right) ;{ }^{1} \mathrm{H} \mathrm{NMR}\left(500 \mathrm{MHz}, \mathrm{CDCl}_{3}\right)$ : $\delta 0.94(\mathrm{~d}, J=7.0 \mathrm{~Hz}, 3 \mathrm{H}), 1.08(\mathrm{~s}, 9 \mathrm{H}), 1.25-$ $1.31(\mathrm{~m}, 4 \mathrm{H}), 1.52(\mathrm{~s}, 3 \mathrm{H}), 1.65-1.68(\mathrm{~m}, 2 \mathrm{H})$, 1.94-1.98 (m, 2H), 2.25-2.35 (m, 2H), 2.43-2.50 $(\mathrm{m}, 1 \mathrm{H}), 3.06(\mathrm{t}, J=10.0 \mathrm{~Hz}, 1 \mathrm{H}), 3.29-3.53(\mathrm{~m}, 1 \mathrm{H})$, 4.30-4.31 (m, 1H), $4.66(\mathrm{~s}, 1 \mathrm{H}), 4.69(\mathrm{~s}, 1 \mathrm{H}), 5.36$ $(\mathrm{m}, 1 \mathrm{H}), 7.35-7.43(\mathrm{~m}, 6 \mathrm{H}), 7.64-7.66(\mathrm{~m}, 4 \mathrm{H})$; ${ }^{13} \mathrm{C}$ NMR (100 MHz, $\left.\mathrm{CDCl}_{3}\right): \delta 14.6,19.6,21.0,22.8$, 23.2, 23.4, 27.3,29.1, 29.3, 29.5, 29.8, 32.5, 40.0, 42.4, $44.4,47.2,60.5,113.1,114.2,127.6,127.7,129.7$, 130.3, 134.2, 134.9, 136.0, 136.1, 145.9; HRMS: calcd for $\mathrm{C}_{31} \mathrm{H}_{42} \mathrm{OSi}[\mathrm{M}+\mathrm{Na}]^{+}$481.2903; found: 481.2905.

2.16 Preparation of $1-(1-((1 R, 2 S, 3 R, 4 S)-3-(($ allyloxy $)$ methyl)-2-methyl-4-(prop-1-en-2-yl)cyclopentyl)-3,3dimethyl-2-phenylbutan-2-yl)benzene (12)

LiHMDS $(0.5 \mathrm{~mL}, 0.50 \mathrm{mmol}, 1 \mathrm{M}$ solution in THF) was added to a stirred solution of alcohol $7(100 \mathrm{mg}$, $0.24 \mathrm{mmol})$ in dry DMF $(3 \mathrm{~mL})$ at $-40^{\circ} \mathrm{C}$ under Argon. After $1 \mathrm{~h}$ of stirring, allyl bromide $(0.05 \mathrm{~mL}$, $0.50 \mathrm{mmol}$ ) was added dropwise. The reaction mixture was stirred for $3 \mathrm{~h}$ and then carefully quenched with saturated aqueous ammonium chloride solution $(2 \mathrm{~mL})$. After removal of most of the solvent under reduced pressure, the resulting residue was extracted with diethyl ether $(4 \times 25 \mathrm{~mL})$. The combined ether extract was successively washed with water $(2 \times 10 \mathrm{~mL})$ and brine $(1 \times 10 \mathrm{~mL})$ and finally dried $\left(\mathrm{Na}_{2} \mathrm{SO}_{4}\right)$. Solvent was removed under reduced pressure and the crude mass obtained was purified by column chromatography over silica gel ( $2 \%$ ethyl acetate in light petroleum) to furnish $12(75 \mathrm{mg}, 69 \%)$ as a viscous liquid. $[\alpha]_{\mathrm{D}}^{25}=$ $7.64\left(\mathrm{c}=1.00, \mathrm{CHCl}_{3}\right) ;{ }^{1} \mathrm{H}$ NMR $\left(400 \mathrm{MHz}, \mathrm{CDCl}_{3}\right)$ :

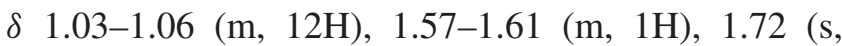
$3 \mathrm{H}), 1.77-1.80(\mathrm{~m}, 1 \mathrm{H}), 2.02-2.04(\mathrm{~m}, 1 \mathrm{H}), 2.11-2.15$ (m, $1 \mathrm{H}), 3.02-3.11(\mathrm{~m}, 2 \mathrm{H}), 3.22(\mathrm{dd}, J=6.4,9.2 \mathrm{~Hz}$, $1 \mathrm{H}), 3.84(\mathrm{t}, J=6.0 \mathrm{~Hz}, 2 \mathrm{H}), 4.24-4.27(\mathrm{~m}, 1 \mathrm{H})$, $4.51(\mathrm{~s}, 1 \mathrm{H}), 4.73(\mathrm{~s}, 1 \mathrm{H}), 5.09(\mathrm{dd}, J=0.8,10.4$ $\mathrm{Hz}, 1 \mathrm{H}), 5.20(\mathrm{dd}, J=1.6,17.6 \mathrm{~Hz}, 1 \mathrm{H}), 5.61-8.85(\mathrm{~m}$, $1 \mathrm{H}), 7.32-7.41(\mathrm{~m}, 6 \mathrm{H}), 7.63-7.67(\mathrm{~m}, 4 \mathrm{H}) ;{ }^{13} \mathrm{C} \mathrm{NMR}$ $\left(100 \mathrm{MHz}, \mathrm{CDCl}_{3}\right): \delta 14.2,15.5,19.6,24.0,27.2,29.1$, 29.3, 29.5, 29.6, 29.7, 39.2, 43.6, 44.8, 46.4, 71.8, 71.9,
$76.3,76.8,110.2,114.2,116.3,127.5,127.6,129.5$, 134.5, 135.1, 135.3, 136.0, 136.1, 146.2; HRMS: calcd for $\mathrm{C}_{29} \mathrm{H}_{40} \mathrm{O}_{2} \mathrm{Si}[\mathrm{M}+\mathrm{Na}]^{+}$471.2695; found: 471.2692.

2.17 Preparation of $(Z, 5 a S, 7 R, 8 S, 8 a R)-3,5 a, 6,7,8,8 a-$ hexahydro-5,8-dimethyl-7-(3,3-dimethyl-2-phenylbutyl)1H-cyclopenta[c]oxepine (13)

To a stirred solution of $\mathbf{1 2}(70 \mathrm{mg}, 0.15 \mathrm{mmol})$ in dry toluene at room temperature under argon atmosphere was added Grubbs second generation catalyst $(12 \mathrm{mg}$, $10 \mathrm{~mol} \%$ ). The reaction mixture was then refluxed for $3 \mathrm{~h}$ and the reaction was monitored by TLC. The solvent was concentrated under vacuum and the resulting residue was purified by column chromatography over silica gel (10\% ethyl acetate in light petroleum) to furnish the cyclized product $13(52 \mathrm{mg}, 78 \%)$. $[\alpha]_{\mathrm{D}}^{25}=$ $14.12\left(\mathrm{c}=0.42, \mathrm{CHCl}_{3}\right) ;{ }^{1} \mathrm{H} \mathrm{NMR}\left(400 \mathrm{MHz}, \mathrm{CDCl}_{3}\right)$ : $\delta 1.01(\mathrm{~d}, J=6.8 \mathrm{~Hz}, 3 \mathrm{H}), 1.06(\mathrm{~s}, 9 \mathrm{H}), 1.55-1.61$ $(\mathrm{m}, 4 \mathrm{H}), 1.81(\mathrm{q}, J=6.8 \mathrm{~Hz}, 1 \mathrm{H}), 1.89-1.94(\mathrm{~m}, 1 \mathrm{H})$, 2.00-2.04 (m, 1H), 2.96-3.04 (m, 1H), 3.62-3.68 (m, $2 \mathrm{H}$ ), 4.12 (appeared as singlet, 2H), 4.20 (appeared as singlet, $1 \mathrm{H}$ ), 5.04 (appeared as singlet, $1 \mathrm{H}$ ), 7.32 $7.42(\mathrm{~m}, 6 \mathrm{H}), 7.66(\mathrm{~d}, J=6.8 \mathrm{~Hz}, 4 \mathrm{H}) ;{ }^{13} \mathrm{C} \mathrm{NMR}$ $\left(75 \mathrm{MHz}, \mathrm{CDCl}_{3}\right): \delta 14.1,19.6,26.9,27.2,30.3,31.5$, $42.9,43.3,43.5,48.6,70.0,70.2,76.6,76.7,120.4$, 127.4, 127.5, 129.6, 134.3. 135.3, 136.0, 136.1, 138.4; HRMS: calcd for $\mathrm{C}_{27} \mathrm{H}_{36} \mathrm{O}_{2} \mathrm{Si}[\mathrm{M}+\mathrm{Na}]^{+}$443.2382; found: 443.2385 .

\section{Results and Discussion}

Thus, the common chiral cyclopentyl aldehyde $\mathbf{8}$ was prepared following the procedure reported ${ }^{12}$ by Ley and co-workers from commercially available S-carvone. The synthesis was initiated by reacting $30 \% \mathrm{H}_{2} \mathrm{O}_{2}$ with $(S)$-carvone 1 to synthesize the epoxide 2 which on trifluoroacetic acid catalyzed the ring opening reaction in the presence lithium chloride furnished the chlorohydrin $\mathbf{3}$ as the only product. The hydroxyl group of $\mathbf{3}$ was then protected as tetrahydropyranyl (THP) ether 4 which on treatment with an ice-cold solution of $\mathrm{NaOMe}$ in methanol afforded the chiral methyl ester $\mathbf{5}$ in good yield (Scheme 2).

The sensitive THP protecting group in $\mathbf{5}$ was transformed to the relatively stable $t$-butyldiphenylsilyl (TBDPS) derivative 6 which on treatment with $\mathrm{LiAlH}_{4}$ furnished the alcohol 7. IBX mediated controlled oxidation of $\mathbf{7}$ afforded the desired chiral aldehyde $\mathbf{8}$ (Scheme 2) in excellent yield. The next target was to convert the aldehyde $\mathbf{8}$ for the required advanced intermediates for fused bicyclic frameworks through RCM reaction. Accordingly, 8 was treated with different 
Grignard reagents made from unsaturated aliphatic bromides to furnish solely the alcohol $\mathbf{9 a}-\mathbf{e}$ as a single isomer which was already explained by Ley et al. ${ }^{12}$ Since, the alcohol 9 was converted immediately to the ketone 10, the detailed study for the stereochemistry of the $-\mathrm{OH}$ group in compound $\mathbf{9}$ was not necessary. Thus, alcohol 9a-e on oxidation with DMP afforded the ketones 10a-e as the sole product in satisfactory yields (Scheme 3).
Now the treatment of the ketones 10a-e with Grubbs-II catalyst $(10 \mathrm{~mol} \%)$ were thoroughly investigated for the synthesis of bicyclic frameworks. Thus, when compound 10a provided the 5-5 fused bicyclic skeleton, 10b and 10c efficiently furnished 5-6 and 5-7 fused bicyclic motifs, respectively, in good yields via ring closing metathesis (RCM) reaction (Scheme 4).

But when compound 10d was subjected to RCM in the presence of Grubbs-II catalyst $(10 \mathrm{~mol} \%)$, it was

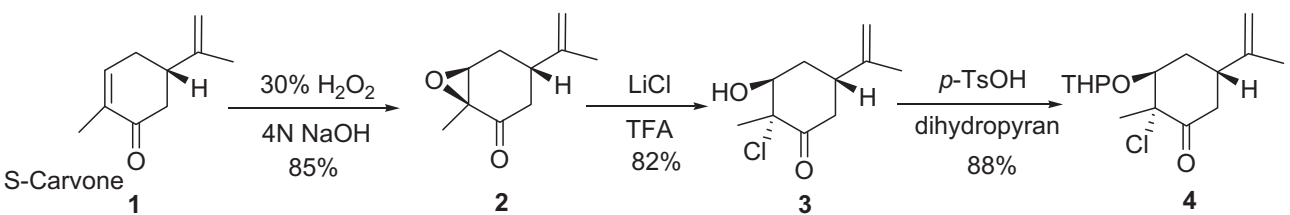

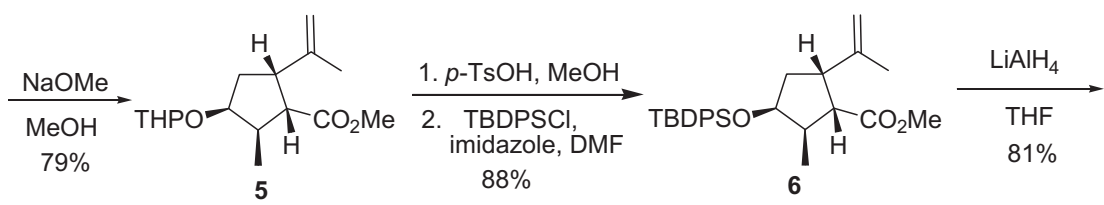

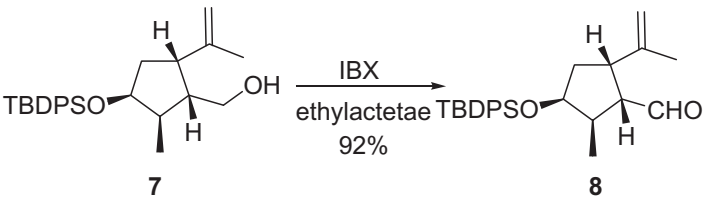

Scheme 2. Synthesis of the known intermediate for the diversity oriented approach.

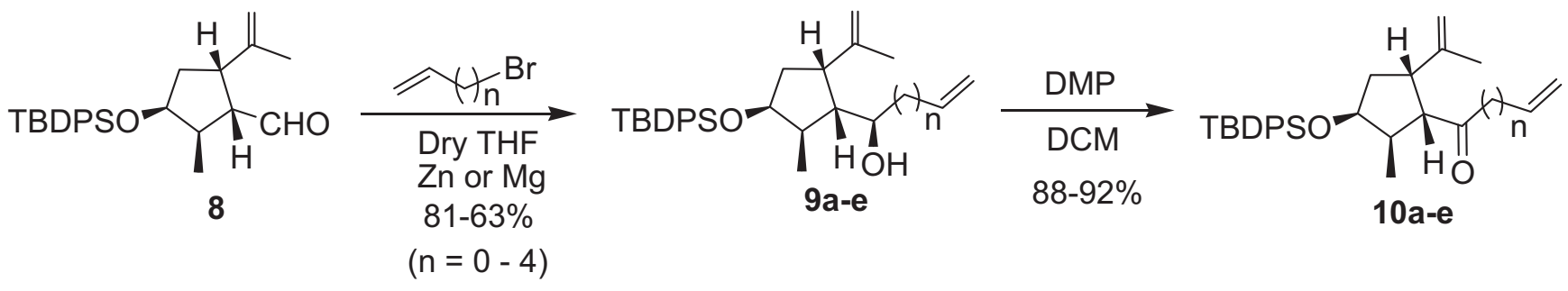

Scheme 3. Synthesis of the precursors for RCM.

a)

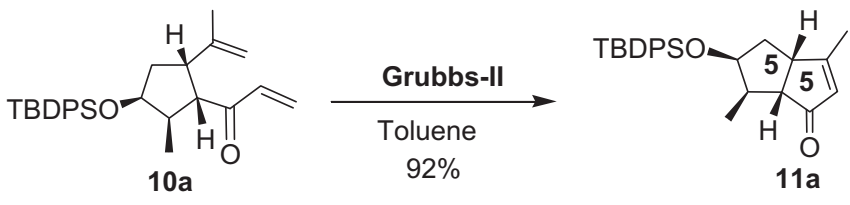

b)
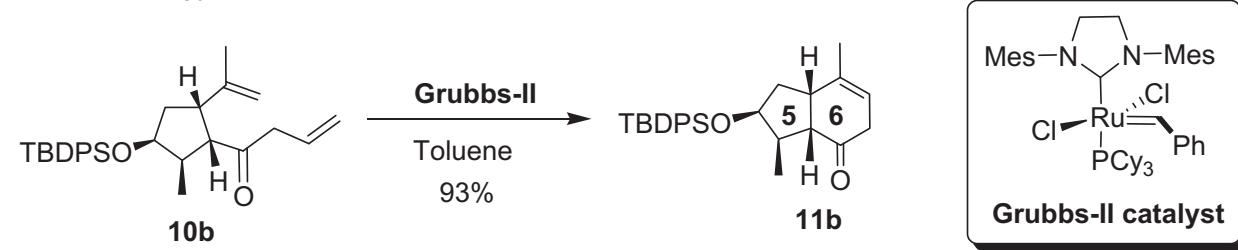

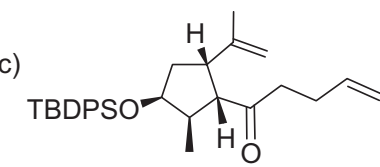

$10 \mathrm{c}$

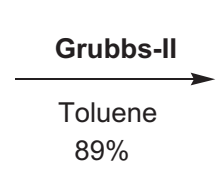

$89 \%$

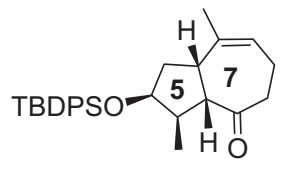

$11 \mathrm{c}$

Scheme 4. Synthesis of 5-5, 5-6 and 5-7 bicyclic frameworks. 
observed that 5-8 fused bicyclic compound 11d (30\%) along with a ring closing carbonyl-olefin metathesis (RCCOM) product 11e (7\%) were formed (Scheme 5). On the other hand, when $20 \mathrm{~mol} \%$ of Grubbs II catalyst was used for RCM, the yield of both 11d (40\%) as well as 11e (13\%) increased which established the concept that ring closing metathesis and ring closing carbonyl-olefin metathesis are very much competitive in nature, so far as the higher-membered ring formation is concerned.

It is already reported ${ }^{11}$ that unlike RCM, the RCCOM propagation requires a stoichiometric amount of Grubbs-II catalyst, as the formation of metal-oxo complex destroys the catalytic activity of Grubbs-II. To extend the protocol for synthesis of 5-9 fused bicyclic carbocycles, compound 10e was treated under identical reaction conditions in the presence of $10 \mathrm{~mol} \%$
Grubbs-II catalyst. It was observed that exclusively the RCCOM product 11f (10\%) could be isolated along with recovery of the starting material 10e (Scheme 6). This result is in accordance with the fact that greater loss of entropy for a nine membered ring formation favored the formation of cyclohexene ring from thermodynamical point of view. The enhancement of the yield of 11f (20\%) with gradual increase of amount of Grubbs-II catalyst $(20 \mathrm{~mol} \%)$, only confirmed the stoichiometric relationship between the substrate and the catalyst.

In this context, it should be mentioned that compared to RCM, available examples of RCCOM are very rare. ${ }^{11}$ Earlier works on RCCOM had involved photochemical reactions, stoichiometric use of metal alkylidene complexes (tungsten, molybdenum and titanium) and use of Tebbe reagents, Petasis reagents and Takai-utimoto

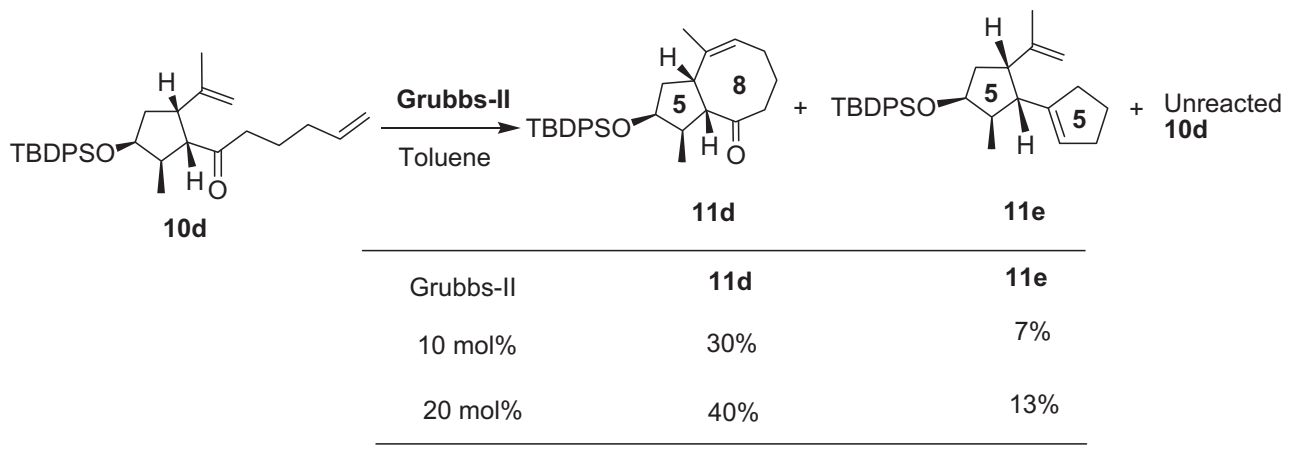

Scheme 5. Grubbs-II catalyzed RCM and RCCOM.

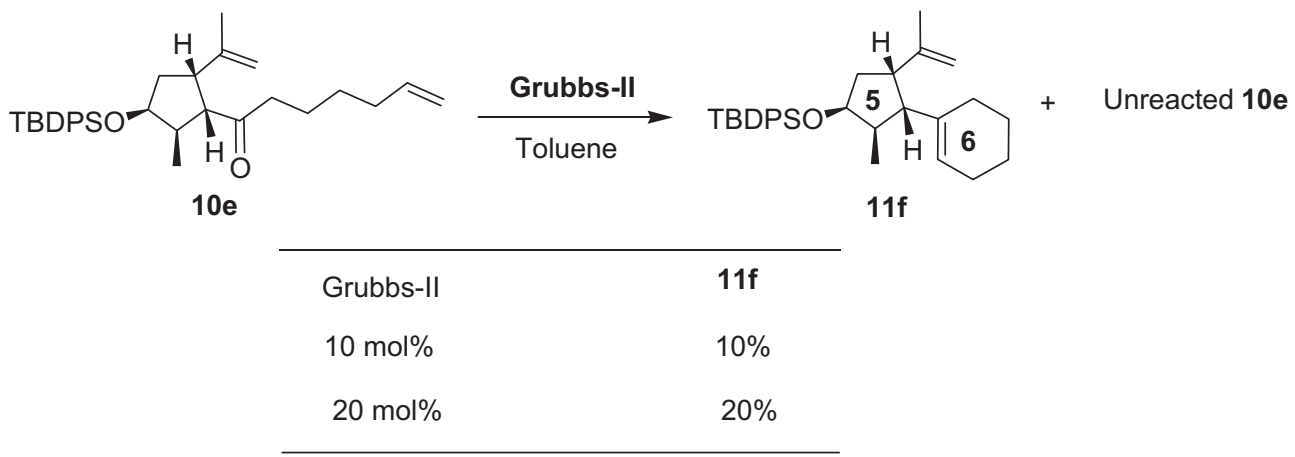

Scheme 6. Grubbs-II catalyzed RCCOM.

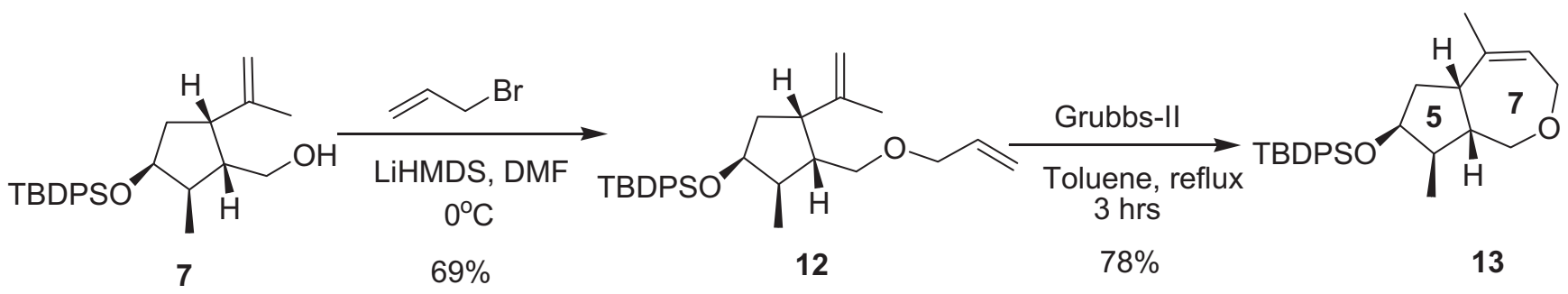

Scheme 7. Synthesis of 5-7 fused oxygen containing heterocycle. 


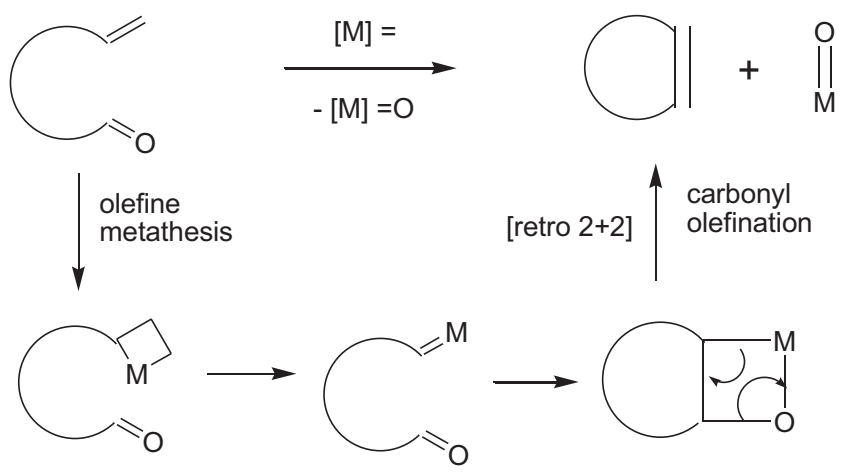

Scheme 8. Probable mechanism for the formation of RCCOM product.

reagents. Very recently, alternative strategies have been envisioned which implicated $\mathrm{FeCl}_{3}$-catalyzed and organocatalytic carbonyl-olefin metathesis methods. ${ }^{13}$ Moreover, there was no indication of $E / Z$ isomerization in final product which is usually a drawback of $\mathrm{RCM}$ for the synthesis of larger rings.

After successful implementation of diversityoriented approach towards the synthesis of a number of bicyclic carbocycles with different ring sizes, we turned our attention for the synthesis of medium-sized cyclopentane-fused oxacyclic skeletons. Such type of oxygen containing heterocycles are primarily found in various biologically important molecules and thus, the developments of new methodologies for synthesizing medium-sized oxacycles have always garnered the attention of scientific community. ${ }^{14}$

Thus, the alcohol 7 on treatment with allyl bromide and LiHMDS in DMF was converted to the allyl ether 12 in good yield (Scheme 7). Compound 12 on ring closing metathesis in the presence of Grubs-II catalyst (10 mol\%) furnished the 5-7 fused oxygen heterocycle $\mathbf{1 3}$ in $78 \%$ yield. The study towards the formation of higher membered oxacylic rings through RCM and their applications for the synthesis of natural products is under progress.

The probable mechanism for the RCCOM reaction, as already indicated by $\mathrm{Fu}$ and Grubbs ${ }^{11}$ with stoichiometric amount of molybdenum alkylidene complex, is depicted below (Scheme 8).

\section{Conclusions}

In summary, diversity-oriented approach for the synthesis of optically active, substituted cyclopentane-fused carbocyclic and oxacyclic frameworks through ring closing metathesis (RCM) from a common chiral aldehyde intermediate prepared from $(S)$-carvone has been demonstrated. As the size of the ring increases, a competition between RCM and ring closing carbonyl-olefin metathesis (RCCOM) was observed. The initial chiral centers have been retained throughout the reaction course. The final compounds have ample scope to be the core structures for biologically active natural products.

\section{Supplementary Information (SI)}

Copies of NMR spectra of new compounds are available in Supplementary Information at www.ias.ac.in/ chemsci.

\section{Acknowledgements}

Authors thank Mr. S Mukherjee, Department of Chemistry, Indian Institute of Technology, Kharagpur, West Bengal 721 302, India and A Chadha, Polyplex Corporation Limited, Sector 1, Noida, Uttar Pradesh 201 301, India for help in the preparation of known starting materials during their (SM and $\mathrm{AC}$ ) summer project training.

\section{References}

1. (a) Burke M D and Schreiber S L 2004 Angew. Chem. Int. Ed. 43 46; (b) Spring D R 2005 Chem. Soc. Rev. 34 472; (c) Tan D S 2005 Nat. Chem. Biol. 1 74; (d) Boethling R S, Sommer E and Di Fiore D 2007 Chem. Rev. 107 2207; (e) Thomas J R and Hergenrother P J 2008 Chem. Rev. 108 1171; (f) Bauer A J and Stockwell B R 2008 Chem. Rev. 108 1774; (g) Schreiber S L 2011 Proc. Natl. Acad. Sci. U.S.A. 1086699 and references cited therein

2. Hoelder S, Clarke P A and Workman P 2012 Mol. Oncol. 6155

3. Shang S and Tan D S 2005 Curr. Opinion Chem. Biol. 3 248; (b) Cordier C, Morton D, Murrison S, Nelson A and O'Leray-Steele C 2008 Nat. Prod. Rep. 4719

4. (a) Devon T K and Scott A I 1972 In Handbook of Naturally Occurring Compounds Vol. II (Academic Press: New York and London); (b) Oishi T, Ohtsuka Y and Rahman A 1989 Stud. Nat. Prod. Chem. 3 73; (c) Faulkner D J 2000 Nat. Prod. Rep. 171 and references cited therein

5. Larry Y 2000 Chem. Rev. 1002963

6. (a) Burke M D and Schreiber S L 2004 Angew. Chem., Int. Ed. 43 46; (b) Thomas G L, Wyatt E E and Spring D R 2006 Curr. Opin. Drug Discovery Dev. 9 700; (c) Nielsen T E and Schreiber S L 2008 Angew. Chem. Int. Ed. 47 48; (d) Spandl R J, Spring D R and Bender A 2008 Org. Biomol. Chem. 61149

7. (a) Chen C, Li X, Neumann C S, Lo M M C and Schreiber S L 2005 Angew. Chem. Int. Ed. 44 2249; (b) Lu K, Huang M, Xiang Z, Liu Y, Chen J and Yang Z 2006 Org. Lett. 8 1193; (c) Kotha S and Singh Kuldeep 2007 Eur. J. Org. Chem. 5909; (d) Spandl R J, Rudyk H and Spring D R 2008 Chem. Commun. 3001; (e) Cordier C, Morton D, Murrison S, Nelson A and O'Leary-Steele C 2008 Nat. Prod. Rep. 25 719; (f) Krishna P R and Reddy P S 2008 J. Comb. Chem. 1 426; (g) Lalli C, 
Trabocchi A, Sladojevich A, Menchi G and Guarna A 2009 Chem. Eur. J. 15 7871; (h) Dandapani S and Marcaurelle L A 2010 Curr. Opin. Chem. Biol. 14 362; (i) Robbins D, Newton A F, Gignoux C, Legeay J-C, Sinclair A, Rejzek M, Laxon C A, Yalamanchili S K, Lewis W, O'Connell M A and Stockman R A 2011 Chem. Sci. 2 2232; (j) Dandapani S, Lowe J T, Comer E and Marcaurelle L A 2011 J. Org. Chem. 76 8042; (k) Hung A W, Ramek A, Wang Y, Kaya T, Wilson J A, Clemons P A and Young D W 2011 Proc. Natl. Acad. Sci. U.S.A. 108 6799; (1) Madsen C M and Clausen M H 2011 Eur. J. Org. Chem. 3107; (m) Dhara K, Midya G C and Dash J 2012 J. Org. Chem. 77 8071; (n) Lenci E, Menchi G, Guarna A and Trabocchi A 2015 J. Org. Chem. 802182

8. (a) Mukherjee P, Roy S J S and Sarkar T K 2010 Org. Lett. 12 2472; (b) Mao B, Fañanás-Mastral M, Lutz M and Feringa B L 2013 Chem. Eur. J. 19761

9. (a) Mehta G and Reddy A V 1981 J. Chem. Soc., Chem. Commun. 756; (b) Huneck S, Baxter G, Cameron A F, Connolly J D and Rycroft D S 1983 Tetrahedron Lett. 24 3787; (c) Paquette L A and Annis G D 1983 J. Am. Chem. Soc. 105 7358; (d) Mehta G, Murthy A N, Reddy D S and Reddy A V 1986 J. Am. Chem. Soc. 108 3443; (e) Harrowven D C, Lucas M C and Howes P D 2000 Tetrahedron Lett. 41 8985; (f) Krafft M E, Cheung Y Y and Abboud K A 2001 J. Org. Chem. 66 7443; (g) Clive D L J, Sannigrahi M and Hisaindee S 2001 J. Org. Chem. 66 954; (h) Winkler J D, Rouse M B, Greaney M F, Harrison S J and Jeon Y T $2002 \mathrm{~J}$. Am. Chem. Soc. 124 9726; (i) Heckrodt T J and Muljer J 2003 J. Am. Chem. Soc. 125 4680; (j) Zanoni G and Franzini M 2004 Angew. Chem. Int. Ed. 43 4837; (k) Miller A K, Hughes C C, Kennedy-Smith J J, Gradl S N and Trauner D 2006 J. Am. Chem. Soc. 128 17057; (1) Pallerla M K and Fox J M 2007 Org. Lett. 9 5625; (m) Clive D L J and Liu D 2008 J. Org. Chem. 73 3078; (n) Chen C-H, Chen Y-K and Sha C-K 2010 Org. Lett. 12 1377; (o) Kim H, Bae H, Kim S, Kim D, Lee D and Paton R S 2011 Tetrahedron 67 10017; (p) Liang Y, Jiang X and
Yu Z-X 2011 Chem. Commun. 47 6659; (q) Tiong E A, Rivalti D, Williams B M and Gleason J L 2013 Angew. Chem. Int. Ed. 52 3442; (r) Jørgensen L, Mckerrall S J, Kuttraff C A, Ungeheuer F, Felding J and Baran P S 2013 Science 341878

10. For selected reviews see: (a) Ivin F J 1983 In Olefin Metathesis (Academic Press: London); (b) Schmalz H G 1995 Angew. Chem. Int. Ed. 34 1833; (c) Grubbs R H, Miller S J and Fu G C 1995 Acc. Chem. Res. 28 446; (d) Grubbs R H and Chang S 1998 Tetrahedron 54 4413; (e) Schuster M and Blechert S 1997 Angew. Chem. Int. Ed. 36 2036; (f) Schrock R R 1999 Tetrahedron 55 8141; (g) Fürstner A 2000 Angew. Chem. Int. Ed. 39 3012; (h) Schrock R R and Hoveyda A H 2003 Angew. Chem. Int. Ed. 42 4592; (i) Deiters A and Martin S F 2004 Chem. Rev. 104 2199; (j) Tori M and Mizutani R 2010 Molecules 15 4242; (k) Blanchard N and Eustache J 2010 In Metathesis in Natural Product Synthesis: Strategies, Substrates and Catalyst (Weinheim: Wiley-VCH)

11. Fu G C and Grubbs R H 1993 J. Am. Chem. Soc. 115 3800

12. (a) Oliver S F, Högenauer K, Simic O, Antonello A, Smith M D and Ley S V 2003 Angew. Chem. Int. Ed. 42 5996; (b) Ley S V, Antonello A, Balskus E P, Booth D T, Christensen S B, Cleator E, Gold H, Högenauer K, Hünger U, Myers R M, Oliver S F, Simic O, Smith M D, Søhoel H and Woolford A J A 2004 Proc. Natl. Acad. Sci. U.S.A. 10112073

13. (a) Griffith A K, Vanos C M and Lambert T H $2012 J$. Am. Chem. Soc. 13418581 and references cited therein; (b) Ludwig J R, Zimmerman P M, Gianino J B and Schindler C S 2016 Nature 533 374; (c) Ma L, Li W, Xi H, Bai X, Ma E, Yan X and Li Z 2016 Angew. Chem. Int. Ed. 5510410

14. For selected reviews on synthesis of oxygen heterocycles, see: (a) Deiters A and Martin S F 2004 Chem. Rev. 104 2199; (b) Grimwood M E and Hansen H C 2009 Tetrahedron 65 8132; (c) Kotha S, Srinivas V and Krishna N G 2012 Heterocycles 861555 